\title{
Path to Overcome Material and Fundamental Obstacles in Spin Valves Based on $\mathrm{MoS}_{2}$ and Other Transition-Metal Dichalcogenides
}

\author{
Marta Galbiati $\odot,{ }^{1,2,{ }^{*}}$ Sergio Tatay, ${ }^{2}$ Simon M.-M. Dubois, ${ }^{1,3}$ Florian Godel, ${ }^{1}$ Regina Galceran, ${ }^{1}$ \\ Samuel Mañas-Valero, ${ }^{2}$ Maëlis Piquemal-Banci, ${ }^{1}$ Aymeric Vecchiola, ${ }^{1}$ Jean-Christophe Charlier, ${ }^{3}$ \\ Alicia Forment-Aliaga, ${ }^{2}$ Eugenio Coronado, ${ }^{2}$ Bruno Dlubak,,${ }^{1, \dagger}$ and Pierre Seneor ${ }^{1, \ddagger}$ \\ 'Unité Mixte de Physique, CNRS, Thales, Univ Paris-Sud, Université Paris-Saclay, 91767 Palaiseau, France \\ ${ }^{2}$ Instituto de Ciencia Molecular, Universitat de València, Catedrático José Beltrán Martínez $n^{\circ} 2$, \\ 46980 Paterna, Spain \\ ${ }^{3}$ Institute of Condensed Matter and Nanosciences (IMCN), Université catholique de Louvain, \\ 1348 Louvain-la-Neuve, Belgium
}

(Received 13 March 2019; revised manuscript received 25 July 2019; published 10 October 2019)

\begin{abstract}
Experimental studies on spin valves with exfoliated 2D materials face the main technological issue of ferromagnetic electrode oxidation during the 2Ds integration process. As a twofold outcome, magnetoresistance (MR) signals are very difficult to obtain and, when they finally are, they are often far from expectations. We propose a fabrication method to circumvent this key issue for 2D-based spintronics devices. We report on the fabrication of $\mathrm{NiFe} / \mathrm{MoS}_{2} / \mathrm{Co}$ spin valves with mechanically exfoliated multilayer $\mathrm{MoS}_{2}$ using an in situ fabrication protocol that allows high-quality nonoxidized interfaces to be maintained between the ferromagnetic electrodes and the 2D layer. Devices display a large MR of 5\%. Beyond interfaces and material quality, we suggest that an overlooked more fundamental physics issue related to spin-current depolarization could explain the limited MR observed so far in $\mathrm{MoS}_{2}$-based magnetic tunnel junctions. This points to a path towards the observation of larger spin signals in line with theoretical predictions above $100 \%$. We envision the impact of our work to be beyond $\mathrm{MoS}_{2}$ and its broader transition-metal dichalcogenides family by opening the way to an accelerated screening of other 2D materials that are yet to be explored for spintronics.
\end{abstract}

DOI: 10.1103/PhysRevApplied.12.044022

\section{INTRODUCTION}

Spintronics is seen as one of the most promising routes for beyond CMOS technology. It aims to enhance electronics with the nonvolatility of magnetism and the additional degree of freedom of spins, giving rise to applications ranging from green electronics with nonvolatile memories to quantum computing [1]. Among all spintronic devices, magnetic tunnel junctions (MTJs) are viewed as the prototypical example [2]. They have already found wide applications, such as magnetic random-access memories (MRAMs) $[3,4]$, magnetic sensors [5,6], or programmable logic devices $[1,7]$. However, until now, all of these

\footnotetext{
*marta.galbiati@uv.es

†bruno.dlubak@cnrs-thales.fr

‡pierre.seneor@cnrs-thales.fr
}

Published by the American Physical Society under the terms of the Creative Commons Attribution 4.0 International license. Further distribution of this work must maintain attribution to the author(s) and the published article's title, journal citation, and DOI. applications have relied mainly on a few materials, $\mathrm{MgO}$ and $\mathrm{Al}_{2} \mathrm{O}_{3}$, as a standard for tunnel barriers in MTJs. Finding suitable barrier layers is still a major challenge. Thus, a consistent technological effort is being made to find alternatives to these materials, not necessarily in terms of magnetoresistance amplitude, but in terms of the precise control of the thickness and homogeneity of the layer, durability, higher temperature stability, or added functionalities. For example, it was demonstrated that new opportunities could be unraveled by interface engineering, thanks to spinterface effects: depending on the ferromagnetic (FM) electrode/barrier coupling at the interface, a spin-polarization enhancement or inversion could be achieved towards increased control over spin-device properties $[8,9]$.

In this scenario, 2D materials have recently emerged as high-potential candidates [10] for use in spin devices, since, thanks to their intrinsic two-dimensional nature, they create extremely thin, tunable, and free from defects barriers with sharp interfaces, which are ideal for spintronic applications. In addition, the blossoming field of 2D materials offers an extremely rich variety of functionalities that cover the full range of electric and magnetic properties: 
from insulators [11-13] to superconductors [14] and from diamagnetic to ferromagnetic $[15,16]$. This makes them perfect candidates for use as building blocks towards the ultimate miniaturization and engineering of spintronic devices, or to be combined in multilayer stacks for the future development of a new generation of spintronic multifunctional devices.

Despite this great potential, investigations into 2D materials for spintronics are still in their infancy. However, first results on graphene implementation in vertical spin valves have already shown promise for spin filtering or as a protective barrier against oxygen $[17,18]$. Other $2 \mathrm{D}$ materials beyond graphene have recently been studied in this context, including hexagonal boron nitride $(h-\mathrm{BN})$ $[19,20], \mathrm{WS}_{2}$ [21], $\mathrm{WSe}_{2}$ [22], black phosphorus (BP) [23], and $\mathrm{MoS}_{2}$ [24-30]. Concerning the large family of transition-metal dichalcogenides (TMDCs), promising theoretical calculations were first reported in 2014 by Dolui et al. [28]. They predicted, by ab initio transport calculations, an extremely large magnetoresistance (MR) signal up to $300 \%$ in $\mathrm{Fe} / \mathrm{MoS}_{2} / \mathrm{Fe}$ MTJs. The first $\mathrm{MoS}_{2}$-based spin valve was experimentally reported soon after in a $\mathrm{NiFe} / \mathrm{MoS}_{2} / \mathrm{NiFe} \mathrm{MTJ}$ [24]. However, although these are pioneering experiments, the observed MR amplitude is well below that of expectations ( $0.73 \%$ of MR signal). A possible explanation is that most experimental studies face the main technological issue of avoiding bottom FM electrode oxidation during the fabrication process, and thus, limiting MR signals. Overall, this leads to the impression that the potential of these materials is poor. Even if recently Khan et al. [27] proposed an in situ flip of the sample to avoid FM electrodes interface oxidation, it appears, overall, that general fabrication procedures to unlock the potential of exfoliated 2D materials for spintronics are still lacking.

Here, we tackle this fundamental problem by developing an easy fabrication approach to incorporate mechanically exfoliated flakes in spintronic devices with high quality and nonoxidized interfaces. This involves the use of an inert atmosphere and $\mathrm{Al}_{2} \mathrm{O}_{3}$ capping layer to avoid bottom FM electrode exposure to air. Motivated by promising theoretical calculations reported in the literature, we report on $\mathrm{MoS}_{2}$ as a prototypical 2D material beyond graphene for MTJs. For this aim, we fabricate $\mathrm{NiFe} / \mathrm{MoS}_{2} / \mathrm{Co}$ spin valves with few-layer-thick $\mathrm{MoS}_{2}$ flakes exfoliated on NiFe. They show MR values well above the current state of the art; thus proving the efficiency of our fabrication method. Moreover, we suggest that an overlooked more fundamental physics issue related to spin-current depolarization could be at the origin of the discrepancy between the large MR signal theoretically predicted in $\mathrm{MoS}_{2}$-based spin valves and the limited spin signal experimentally observed in these devices; thus renewing interest in $\mathrm{MoS}_{2}$ for spintronic devices and indicating a path for their further development towards applications.

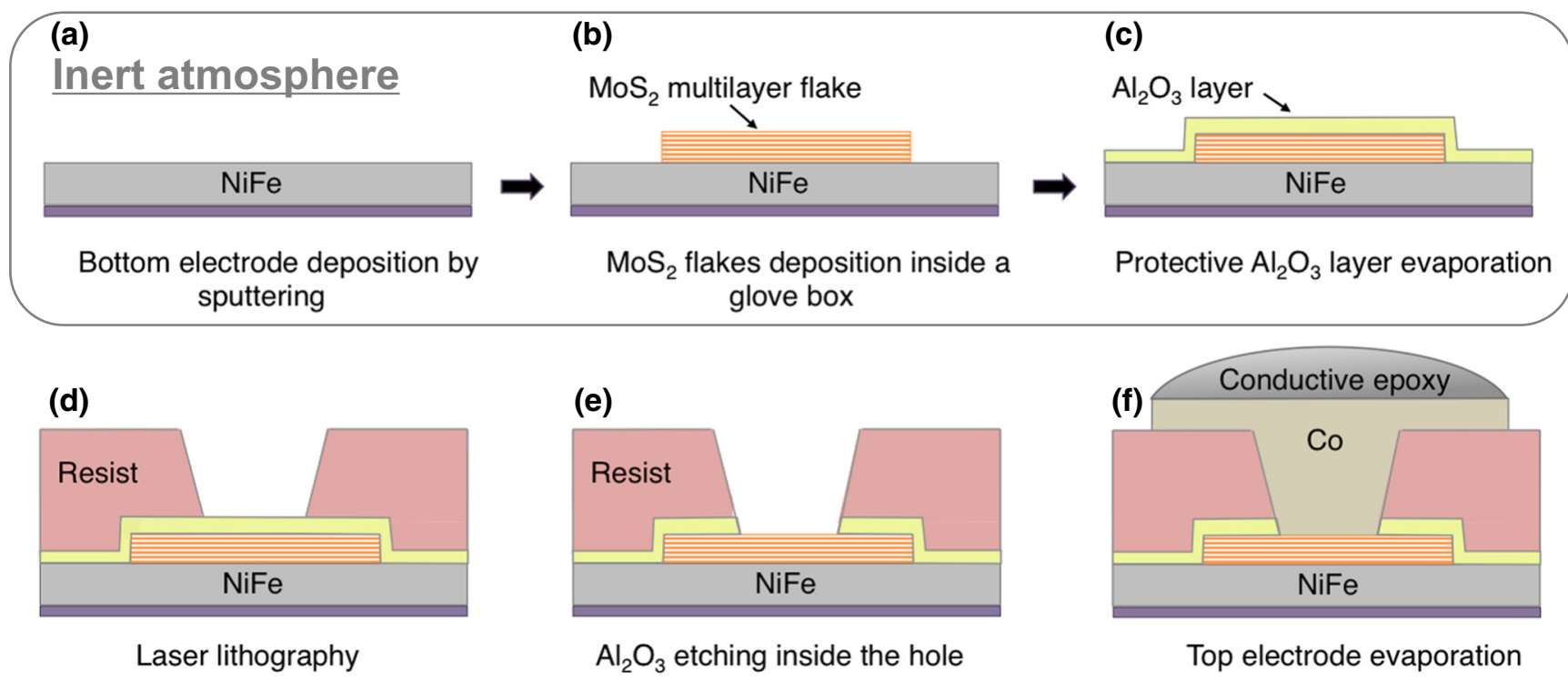

FIG. 1. Schematic of device fabrication process. (a) A NiFe layer is deposited over a $\mathrm{Si} / \mathrm{SiO}_{2}$ substrate by sputtering and transferred under vacuum into a glove box. (b) $\mathrm{MoS}_{2}$ flakes are mechanically exfoliated inside the glove box and deposited over the sample. (c) Sample is transferred into an evaporator under a controlled atmosphere, where a $1 \mathrm{~nm}$ Al protective layer is evaporated over the whole sample and then oxidized in air. (d) Laser lithography is used to open a hole of 1-2 $\mu \mathrm{m}$ in diameter over the selected flakes, and (e) $\mathrm{Al}_{2} \mathrm{O}_{3}$ layer is removed inside the hole. (f) Device is completed with evaporation of the top electrode, Co $(20 \mathrm{~nm}) / \mathrm{Au}(80 \mathrm{~nm})$, and a conductive epoxy drop is deposited on each contact to protect them during the ion beam etching (IBE) process used to electrically isolate the junction. 
(a)

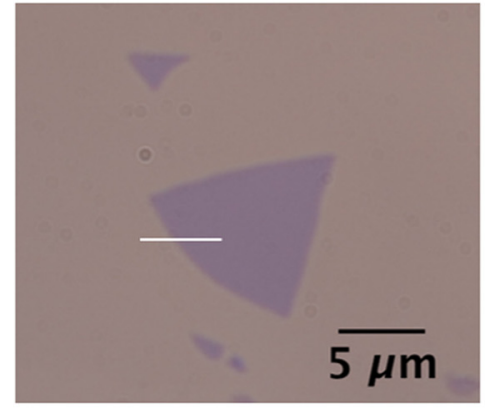

(b)

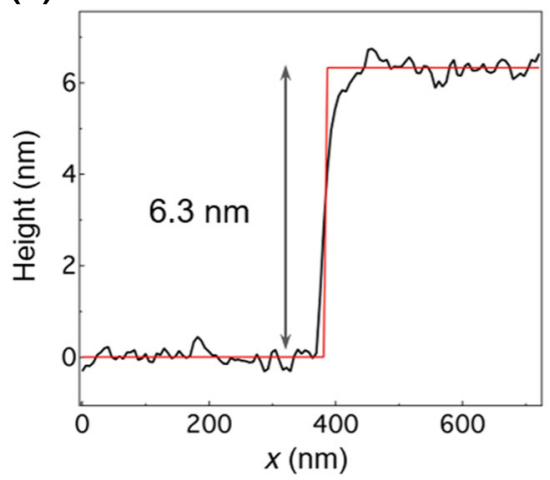

(c)

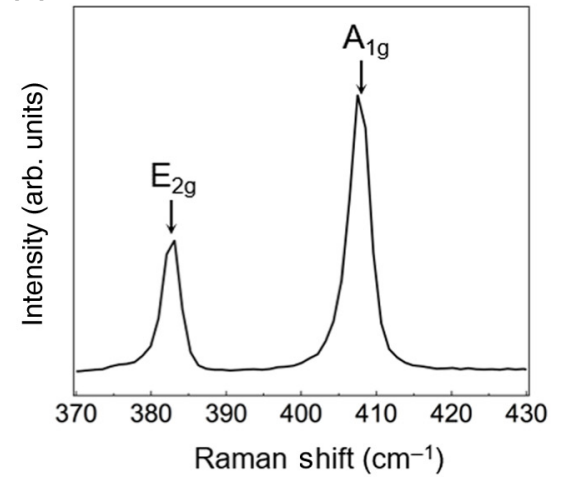

FIG. 2. (a) Optical microscope image of a $\mathrm{MoS}_{2}$ flake deposited over the NiFe electrode and covered with the $\mathrm{Al}_{2} \mathrm{O}_{3}$ layer. (b) $\mathrm{Height}$ profile of the flake shown in (a) measured by atomic force microscopy (AFM) in tapping mode. (c) Raman spectrum measured on the flake with an excitation laser of $514 \mathrm{~nm}$.

\section{FABRICATION METHOD}

The device fabrication procedure is illustrated in Fig. 1. First, a $\mathrm{NiFe}(15 \mathrm{~nm})$ layer is deposited by magnetron sputtering on a $\mathrm{Si} / \mathrm{SiO}_{2}$ substrate in a high-vacuum (HV) chamber, with a base pressure of $10^{-7}$ mbar [Fig. 1(a)]. Then, we use a dedicated vacuum-sealed chamber to directly transfer the sample from the deposition chamber to a glove box without breaking the vacuum. A MoS 2 crystal (from Manchester Nanomaterials Company) is mechanically exfoliated inside the glove box using the Scotch-tape technique and $\mathrm{MoS}_{2}$ flakes are thus deposited on the $\mathrm{NiFe}$ surface [Fig. 1(b)]. At this point, the sample is transferred again through the sealed chamber under a controlled atmosphere from the glove box to a HV e-beam evaporator. A thin Al layer of $1 \mathrm{~nm}$ is evaporated on the whole surface and then oxidized in an ambient atmosphere to form a thin $\mathrm{Al}_{2} \mathrm{O}_{3}$ protective barrier [Fig. 1(c)]. Once capped, the sample can be exposed to the air without bottom FM electrode oxidation. Thin $\mathrm{MoS}_{2}$ layers are still visible through the $\mathrm{Al}_{2} \mathrm{O}_{3}$ barrier and an optical microscope is used to select $\mathrm{MoS}_{2}$ flakes over the sample [Fig. 2(a)]. Their thickness is then characterized by AFM, as shown in Fig. 2(b), and Raman spectroscopy is used to demonstrate their structural quality. Fig. 2(c) displays the two Raman-active modes at about $384 \mathrm{~cm}^{-1}\left(\mathrm{E}_{2 \mathrm{~g}}\right)$ and $\sim 407 \mathrm{~cm}^{-1}\left(\mathrm{~A}_{1 \mathrm{~g}}\right)$, which are measured at room temperature on the flake with an excitation laser of $514 \mathrm{~nm}$. Among the exfoliated flakes, we select multilayers of about 5-7 $\mathrm{nm}$. This choice is motivated by theoretical calculations that predict a significant increase in the MR signal from that expected in junctions incorporating a monolayer to junctions incorporating flakes thicker than three layers [28]. Indeed, from fully atomistic first-principles transport calculations it is predicted that, while thin layers are metallic, in thicker junctions, the native gap of $\mathrm{MoS}_{2}$ is expected to reemerge, leading to a MR increase, which saturates for the thickest junctions calculated for nine layers. In the case of the flake shown in Fig. 2, its thickness is around $6.3 \mathrm{~nm}$, which corresponds to nine layers.

After this characterization of the flakes, samples are spin-coated with UVIII photoresist $(\approx 800 \mathrm{~nm}$ thick) and a laser lithography process is used to open holes of 1-2 $\mu \mathrm{m}$ in diameter in the resist over the selected flakes [Fig. 1(d)]. At this point, the $\mathrm{Al}_{2} \mathrm{O}_{3}$ protection layer is removed only inside the opened holes using resist developer to allow direct contact on the flake [Fig. 1(e)]. Finally, the device is completed with e-beam evaporation of the top Co $(20 \mathrm{~nm}) / \mathrm{Au}(80 \mathrm{~nm})$ electrode. A conductive epoxy drop is manually deposited over each contact to protect them during the IBE process used to electrically isolate the junction. The structure of the final device is shown in Fig. 1(f).

The fabricated $\mathrm{NiFe} / \mathrm{MoS}_{2} / \mathrm{Co}$ junctions are measured inside a cryostat by applying an external in-plane magnetic field [schematic of the junction in Fig. 3(a)].

\section{RESULTS AND DISCUSSION}

As shown in Fig. 3(b), a clear positive MR signal can be observed at low temperature in a $\mathrm{NiFe} / \mathrm{MoS}_{2} /$ Co junction with a flake thickness of $6.3 \mathrm{~nm}$. This value is calculated as $\mathrm{MR}=\left(\left(R_{\mathrm{AP}}-R_{P}\right) / R_{P}\right) \times 100$, where $R_{\mathrm{AP}}$ and $R_{P}$ are the resistances of the device in the antiparallel and parallel spin configurations of the electrodes, respectively. The first switch is observed at a coercive field of about $\pm 8 \mathrm{mT}$ and presents a small asymmetry. This can be ascribed to a smooth switch of NiFe, as the bottom electrode is large and probably multidomain, presenting a fast nucleationpropagation switching mechanism. Co switch is observed at around $\pm 35 \mathrm{mT}$. Because the surface of the top electrode is smaller and probably monodomain, the switching mechanism is controlled by shape anisotropy, which makes a more abrupt switch and influences the coercive field. Remarkably, as reported in Fig. 3, a MR signal of up to $5 \%$ is observed in this junction. This signal is well above 
(a)
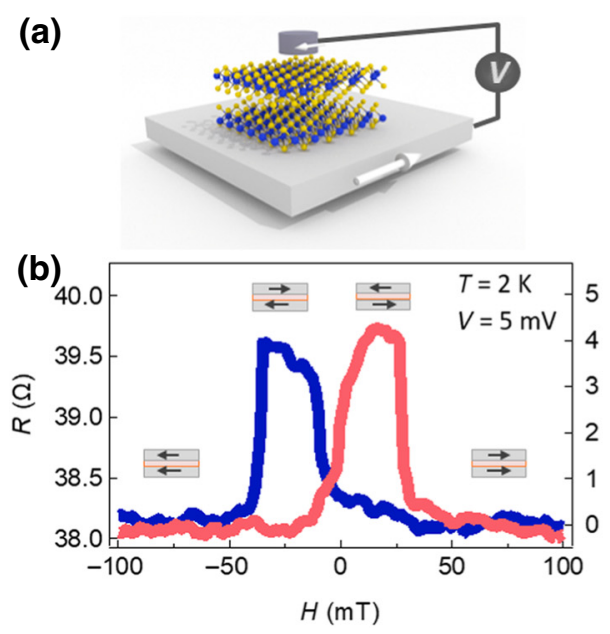

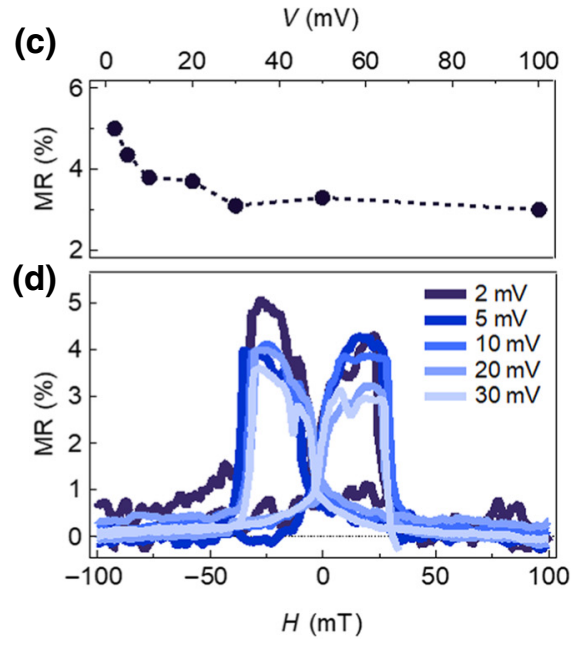

FIG. 3. (a) Schematic of the measured $\mathrm{NiFe} / \mathrm{MoS}_{2} / \mathrm{Co}$ device. (b) MR signal is measured in a junction with flake thickness of $6.3 \mathrm{~nm}$. Signal is measured at $2 \mathrm{~K}$ and for an applied bias of $5 \mathrm{mV}$. (c) MR dependence with applied bias voltage measured at $2 \mathrm{~K}$ on the same junction. Dotted line is a guide to the eye. (d) MR curves measured a $2 \mathrm{~K}$ for different bias voltages. results reported in the literature for $\mathrm{MoS}_{2}$-based (and more generally TMDC-based) MTJs. This positive result thus validates our particular in situ fabrication approach. Figures 3(c) and 3(d) also report the MR behavior with bias voltage. We observe a monotonous decrease in the MR signal when increasing the bias voltage, which corresponds to conventional behavior usually attributed to $s$-like carriers [31].

Surprisingly, metallic behavior is observed, even in these junctions incorporating flakes of about $6 \mathrm{~nm}$ thick. This behavior is already reported in $\mathrm{MoS}_{2}$-based MTJs with thinner layers [24]; transition-metal/ $\mathrm{MoS}_{2}$ interfaces $[32,33]$; and in junctions incorporating other $2 \mathrm{D}$ materials, such as $\mathrm{WSe}_{2}$ [22] or BP [23] with layers of 5-6.5 nm thick, or even in wide band gap h-BN [34]. We show, in our first-principle calculations (see Fig. 4), that the first $\mathrm{MoS}_{2}$ layer bound to the Co contact becomes metallic due to hybridization between Co and S, leaving the second one semiconducting. The carriers easily flow laterally within the first layer, while the presence of impurities and absorbates acting as dopants in $\mathrm{MoS}_{2}$ contribute to this metallic behavior in the region outside the contact
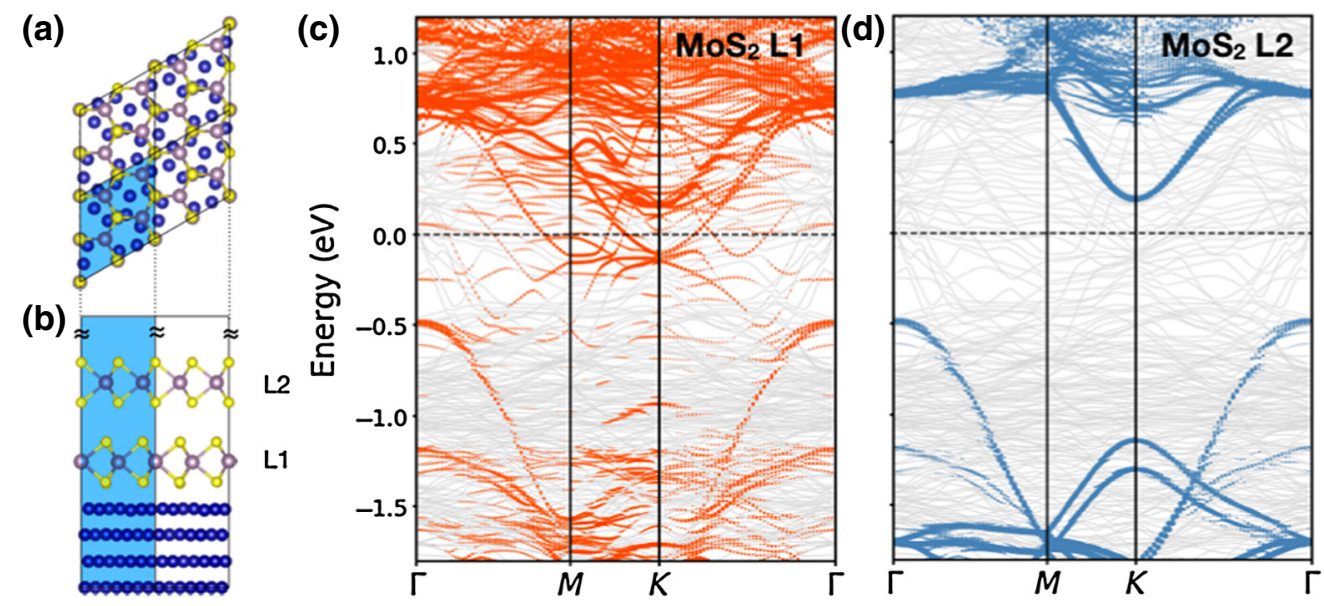

FIG. 4. Electronic band structure of $\mathrm{Co} / \mathrm{MoS}_{2}$ bilayer interface, highlighting the impact of hybridization. (a), (b) Ball and stick representation of the computed $\mathrm{Co} / \mathrm{MoS}_{2}$ bilayer interface. Top and side views are depicted in (a) and (b), respectively. The periodic computational unit cell is highlighted in blue. (c), (d) Electronic band structure of the heterostructure computed in the framework of spin-polarized density functional theory, including spin-orbit coupling. The full set of electronic bands (including the metal) is displayed in gray. The red (blue) dots correspond to the projection of the Hamiltonian eigenstates onto the Mo and $\mathrm{S}$ atoms of the first (second) $\mathrm{MoS}_{2}$ layer labeled L1 (L2) in (b). The size of the dot is proportional to the amplitude of the projection, i.e., the "MoS 2 " character of the eigenstate. The Fermi level of the interface is used as energy reference and depicted as a horizontal dashed line. These results are obtained using the projector augmented wave method and the Perdew-Burke-Ernzenhofer (PBE) parametrization of the generalized gradient approximation (GGA), as implemented within Vienna Ab Initio Simulation Package (VASP) [40-43]. An energy cutoff of $400 \mathrm{eV}$ and a $8 \times 8 \times 1$ gamma-centered Monkhorst-Pack grid are used to compute the self-consistent electronic density and to relax the atomic positions up to forces lower than $5 \times 10^{-5} \mathrm{eV} / \mathrm{A}$. 
(a)

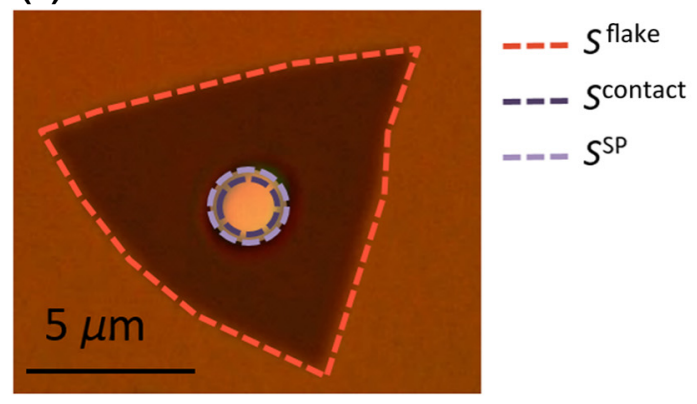

(b)

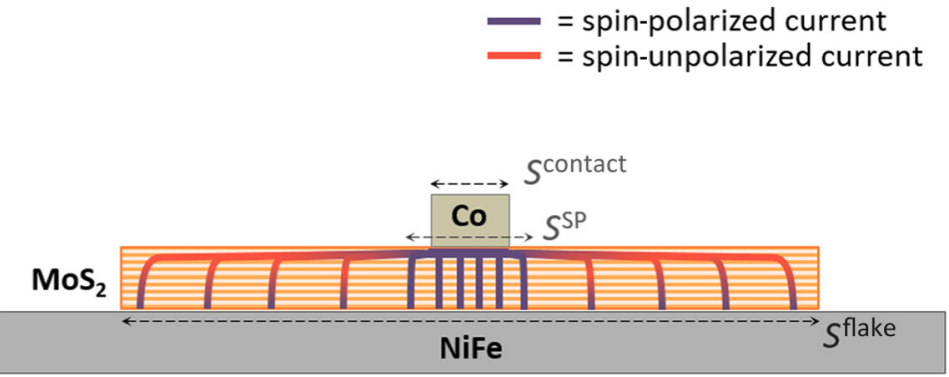

FIG. 5. (a) Optical microscope image of the flake measured in the junction after the lithography process. From this image, the total surface of the flake is measured to be $59 \mu \mathrm{m}^{2}$ and the contact diameter $L=1.5 \mu \mathrm{m}$. (b) Schematic of transport behavior through the flake. Charges injected in the junction are delocalized over the whole flake due to the large lateral transport contribution in $\mathrm{MoS}_{2}$. However, spin-polarized charges are only in a small area around the contact $\left(S_{\mathrm{SP}}=3.14 \mu \mathrm{m}^{2}\right)$, while the rest of the charges are not spin-polarized.

$[33,35,36]$. To a lesser extent, the same type of in-plane to out-of-plane conductivity imbalance is expected in the next layers because intralayer transport in $\mathrm{MoS}_{2}$ is much higher than that of interlayer transport [37,38]. This is acknowledged by our recent conducting tip (CT) AFM local measurements on $\mathrm{MoS}_{2}$ flakes and demonstrates that charges are completely delocalized on all our interconnected layers, i.e., a strong lateral transport contribution must be considered [39].

This raises an issue that is highly relevant, when targeting applications, and is typical of most devices based on (semi) conducting flakes of 2D materials. Indeed, in these devices, the barrier material (i.e., the flake) has a larger surface than that of the contact, $S_{\text {contact. }}$ When this material becomes conductive, the carriers can become delocalized over the whole flake surface, $S_{\text {flake }}$ (as shown in Fig. 5). As the injected spin polarization decays exponentially away from the contact, the spin-polarized current, $I_{\mathrm{SP}}$, then only represents a fraction of the total flake current, $I_{\text {total }}$. Thus, the measured magnetoresistance,

$$
\mathrm{MR}_{\text {meas }}=\frac{I_{\mathrm{AP}}-I_{P}}{I_{\mathrm{AP}}}=\frac{\Delta I_{\mathrm{SP}}}{I_{\text {total }}}=\eta \frac{\Delta I_{\mathrm{SP}}}{I_{\mathrm{SP}}},
$$

corresponds only to a fraction, $\eta$, of the ideal $\mathrm{MR}=$ $\Delta I_{\mathrm{SP}} / I_{\mathrm{SP}}$ that would be expected for a perfect junction limited to the spin-polarized area. Here, with $l_{\text {sf }}$ as the spindiffusion length in the barrier and $L$ as the contact diameter, $\eta$ is expected to range from $\eta=1$ if $l_{\text {sf }} \gg L$ to $\eta=$ $S_{\text {contact }} / S_{\text {flake }}$, when $l_{\text {sf }} \ll L$, and the current can be considered as spin-polarized only in the region corresponding to the contact. As an example, for our device, we find $L=$ $1.5 \mu \mathrm{m}$ and $S_{\text {flake }}=59 \mu \mathrm{m}^{2}$ for the whole flake surface [Fig. 5(a)]. Considering $l_{\mathrm{sf}}=235 \mathrm{~nm}$ reported for $\mathrm{MoS}_{2}$ [44], in a first approximation, we can assume $l_{\text {sf }} \ll L$. This leads to $\eta=0.03$ and a corresponding MR $>160 \%$. If we include the whole area within the spin-diffusion length, $l_{\text {sf }}$, from the contact, we can estimate the effective area of spin injection to be $S_{\mathrm{SP}}=\pi\left(L / 2+l_{\mathrm{sf}}\right)^{2}=3.14 \mu \mathrm{m}^{2}$ for our device, which leads to $\eta=S_{\mathrm{SP}} / S_{\text {flake }}=0.053$. Even in this less favorable case, the estimated MR for the contact is still found to be MR $=94 \%$.

This high MR is in line with the expected quality of the interfaces achieved with our process and suggests that the full potential of $\mathrm{MoS}_{2}$ may be soon demonstrated in spintronics devices. Indeed, our findings show that results on $\mathrm{MoS}_{2}$ (but also other TMDCs and semiconducting 2Ds) may have been hampered by a fundamental spindepolarization obstacle, in addition to the commonly raised material and interface quality issues. This may also explain why reported MR signals are still far from that of theoretical expectations. Importantly, this obstacle should be addressable through relevant device design and fabrication technology, giving a path towards 2D materials integration into 2D MTJ applications.

Still, we cannot rule out the possibility that the current propagating laterally in the top layer of $\mathrm{MoS}_{2}$ remains spin-polarized over the whole flake; this scenario would reveal $\mathrm{MoS}_{2}$ to be highly suitable for use as a conductive channel for spin transport in spin-FET devices. Notably, however, this situation would require spin-diffusion lengths well above $5 \mu \mathrm{m}$, which would be much higher than the value of $235 \mathrm{~nm}$ reported until now in the literature.

\section{CONCLUSION}

Here, we demonstrate the successful fabrication of $\mathrm{NiFe} / \mathrm{MoS}_{2} / \mathrm{Co}$ spin valves incorporating few-layered $\mathrm{MoS}_{2}$. The original approach we adopt involves a passivation scheme. This procedure proves to be extremely effective for obtaining high-quality nonoxidized interfaces. Indeed, a strong improvement in the magnitude of the MR signal is observed in our devices, compared with results reported in the literature. By circumventing the key oxidation issue for spintronics devices, these results should 
unlock the possibility to tune spin-device barriers and potentially incorporate every $2 \mathrm{D}$ material through the same process. This would greatly accelerate their screening and allow investigations into a plethora of new 2D materials for spintronics. Moreover, the simple model we suggest highlights what could be the overlooked relevant physical mechanisms at play in these kinds of devices. This allows to estimate that a much higher MR signal (up to about $94 \%$ in our devices) could be expected in $\mathrm{MoS}_{2}$-based junctions, if the whole surface of the flake is active with a spinpolarized current. Showing that there is a material and, more importantly, a physics issue, our findings strengthen the potential of $2 \mathrm{D}$ materials for spintronics and highlight a way to go beyond the limited spin-signal results obtained, to date, for $\mathrm{MoS}_{2}$-based MTJs. Thus, we believe it will be a useful guide towards the improvement of devices based on TMDCs, such as $\mathrm{MoS}_{2}$ and beyond.

\section{ACKNOWLEDGMENTS}

We acknowledge financial support from the European Commission through H2020 Future and Emerging Technologies Graphene Flagship (Grants No. 696656 and No. 785219), 2D-INK (Grant No. 664878), H2020 European Research Council Advanced Grant Mol-2D (Grant No. 788222), European Cooperation in Science and Technology MOLSPIN (Grant No. CA15128), H2020 Marie Skłodowska-Curie Actions SPIN2D (Grant No. H2020/2014-659378) to M.G. and ITN Spinograph (Grant No. 607904) to R.G., Spanish MINECO (Grant No. MAT2017-89993-R cofinanced by FEDER and Excellence Unit "Maria de Maeztu" Grant No. MDM-2015-0538), and the Generalitat Valenciana (Prometeo Program/2017/066). S.M.-V. thanks the Spanish MECD for support from the Formación de Profesorado Universitario Program (Grant No. FPU014/04407). S.M.-M.D. thanks the National Fund for Scientific Research of Belgium [F.R.S.- FNRS] and the Fédération Wallonie-Bruxelles through the "3D nanoarchitecturing of 2D crystals" (Grant No. ARC - 16/21-077). Computational resources were provided by the supercomputing facilities of the Universitee catholique de Louvain (CISM/UCL) and the "Consortium des Equipements de Calcul Intensif” en Fédération Wallonie-Bruxelles (CECI). The authors are also grateful to E. Tormos-Feliu, A. LopezMuñoz, J.M. Martínez Agudo, and S. Delprat for their technical support.

[1] B. Behin-Aein, D. Datta, S. Salahuddin, and S. Datta, Proposal for an all-spin logic device with built-in memory, Nat. Nanotechnol. 5, 266 (2010).

[2] M. Julliere, Tunneling between ferromagnetic films, Phys. Lett. A 54, 225 (1975).

[3] E. Chen, D. Apalkov, Z. Diao, D. Druist, D. Lottis, V. Nikitin, X. Tang, S. Watts, S. Wang, S. A. Wolf, A. W.
Ghosh, J. W. Lu, S. J. Poon, M. Stan, W. H. Butler, S. Gupta, C. K. A. Mewes, T. Mewes, and P. B. Visscher, Advances and future prospects of spin-transfer torque random access memory, IEEE Trans. Magn. 46, 1873 (2010).

[4] C. Chappert, A. Fert, and F. Nguyen Van Dau, The emergence of spin electronics in data storage, Nat. Mater. 6, 813 (2007).

[5] J. R. Childress and R. E. Fontana, Magnetic recording read head sensor technology, C. R. Phys. 6, 997 (2005).

[6] J.-G. Zhu and C. Park, Magnetic tunnel junctions, Mater. Today 9, 36 (2006).

[7] H. Dery, P. Dalal, L. Cywinski, and L. J. Sham, Spin-based logic in semiconductors for reconfigurable large-scale circuits, Nature 447, 573 (2007).

[8] C. Barraud, P. Seneor, R. Mattana, S. Fusil, K. Bouzehouane, C. Deranlot, P. Graziosi, L. Hueso, I. Bergenti, V. Dediu, F. Petroff, and A. Fert, Unravelling the role of the interface for spin injection into organic semiconductors, Nat. Phys. 6, 615 (2010).

[9] M. Galbiati, S. Tatay, C. Barraud, A. V. Dediu, F. Petroff, R. Mattana, and P. Seneor, Spinterface: Crafting spintronics at the molecular scale, MRS Bull. 39, 602 (2014).

[10] M. Piquemal-Banci, R. Galceran, M. Martin, F. Godel, A. Anane, F. Petroff, B. Dlubak, and P. Seneor, 2D-MTJs: introducing 2D materials in magnetic tunnel junctions, J. Phys. D: Appl. Phys. 50, 203002 (2017).

[11] L. A. Ponomarenko, A. K. Geim, A. A. Zhukov, R. Jalil, S. V. Morozov, K. S. Novoselov, I. V. Grigorieva, E. H. Hill, V. V. Cheianov, V. I. Fal, K. Watanabe, T. Taniguchi, and R. V. Gorbachev, Tunable metal-insulator transition in double-layer graphene heterostructures, Nat. Phys. 7, 958 (2011).

[12] G.-H. Lee, Y. Yu, C. Lee, C. Dean, K. L. Shepard, G. Lee, Y. Yu, C. Lee, C. Dean, K. L. Shepard, P. Kim, and J. Hone, Electron tunneling through atomically flat and ultrathin hexagonal boron nitride, Appl. Phys. Lett. 99, 243114 (2011).

[13] D. Golberg, Y. Bando, Y. Huang, T. Terao, M. Mitome, and C. Tang, Boron nitride nanotubes and nanosheets, ACS Nano 4, 2979 (2010).

[14] B. Sipos, A. F. Kusmartseva, A. Akrap, H. Berger, L. Forro, and E. Tutis, From Mott state to superconductivity in 1TTaS2, Nat. Mater. 7, 960 (2008).

[15] H. Li, X. Qi, J. Wu, Z. Zeng, J. Wei, H. Zhang, M. Science, N. Avenue, M. Technology, and N. Drive, Investigation of $\mathrm{MoS}_{2}$ and graphene nanosheets by magnetic force microscopy, ACS Nano 7, 2842 (2013).

[16] B. Huang, G. Clark, E. Navarro-Moratalla, D. R. Klein, R. Cheng, K. L. Seyler, D. Zhong, E. Schmidgall, M. A. McGuire, D. H. Cobden, W. Yao, D. Xiao, P. JarilloHerrero, and X. Xu, Layer-dependent ferromagnetism in a van der Waals crystal down to the monolayer limit, Nature 546, 270 (2017).

[17] M.-B. Martin, B. Dlubak, R. S. Weatherup, H. Yang, C. Deranlot, K. Bouzehouane, F. Petroff, A. Anane, S. Hofmann, J. Robertson, A. Fert, and P. Seneor, Sub-nanometer atomic layer deposition for spintronics in magnetic tunnel junctions based on graphene spin-filtering membranes, ACS Nano 8, 7890 (2014). 
[18] M. B. Martin, B. Dlubak, R. S. Weatherup, H. Yang, R. Blume, R. Schloegl, S. Collin, S. Hofmann, J. Robertson, A. Anane, A. Fert, and P. Seneor, Protecting nickel with graphene spin-filtering membranes: A single layer is enough, Appl. Phys. Lett. 107, 012408 (2015).

[19] M. Piquemal-Banci, R. Galceran, S. Caneva, M.-B. Martin, R. S. Weatherup, P. R. Kidambi, K. Bouzehouane, S. Xavier, A. Anane, F. Petroff, A. Fert, J. Robertson, S. Hofmann, B. Dlubak, and P. Seneor, Magnetic tunnel junctions with monolayer hexagonal boron nitride tunnel barriers, Appl. Phys. Lett. 108, 102404 (2016).

[20] A. Dankert, M. Venkata Kamalakar, A. Wajid, R. S. Patel, S. P. Dash, M. V. Kamalakar, A. Wajid, R. S. Patel, and S. P. Dash, Tunnel magnetoresistance with atomically thin twodimensional hexagonal boron nitride barriers, Nano Res. 8, 1357 (2015).

[21] M. Z. Iqbal, M. W. Iqbal, S. Siddique, M. F. Khan, and S. M. Ramay, Room temperature spin valve effect in $\mathrm{NiFe} / \mathrm{WS}_{2} /$ Co junctions, Sci. Rep. 6, 21038 (2016).

[22] K. Zhao, Y. Xing, J. Han, J. Feng, W. Shi, B. Zhang, and Z. Zeng, Magnetic transport property of $\mathrm{NiFe} / \mathrm{WSe}_{2} / \mathrm{NiFe}$ spin valve structure, J. Magn. Magn. Mater. 432, 10 (2017).

[23] L. Xu, J. Feng, K. Zhao, W. Lv, X. Han, Z. Liu, X. $\mathrm{Xu}, \mathrm{H}$. Huang, and Z. Zeng, Magnetoresistance effect in $\mathrm{NiFe} / \mathrm{BP} / \mathrm{NiFe}$ vertical spin valve devices, Adv. Condens. Matter Phys. 2017, 9042823 (2017).

[24] W. Wang, A. Narayan, L. Tang, K. Dolui, Y. Liu, X. Yuan, Y. Jin, Y. Wu, I. Rungger, S. Sanvito, and F. Xiu, Spin-valve effect in $\mathrm{NiFe} / \mathrm{MoS}_{2} / \mathrm{NiFe}$ junctions, Nano Lett. 15, 5261 (2015).

[25] A. Dankert, P. Pashaei, M. V. Kamalakar, A. P. S. Gaur, S. Sahoo, I. Rungger, A. Narayan, K. Dolui, M. A. Hoque, R. S. Patel, M. P. de Jong, R. S. Katiyar, S. Sanvito, and S. P. Dash, Spin-polarized tunneling through chemical vapor deposited multilayer molybdenum disulfide, ACS Nano 11, 6389 (2017).

[26] W. C. Wong, S. M. Ng, H. F. Wong, C. L. Mak, and C. W. Leung, Spin-valve junction with transfer-free $\mathrm{MoS}_{2}$ spacer prepared by sputtering, IEEE Trans. Magn. 53, 1600205 (2017).

[27] M. F. Khan, H. Kim, G. Nazir, S. Jung, and J. Eom, Layer dependent magnetoresistance of vertical $\mathrm{MoS}_{2}$ magnetic tunnel junctions, Nanoscale 10, 16703 (2018).

[28] K. Dolui, A. Narayan, I. Rungger, and S. Sanvito, Efficient spin injection and giant magnetoresistance in $\mathrm{Fe} / \mathrm{MoS}_{2} / \mathrm{Fe}$ junction, Phys. Rev. B 90, 041401(R) (2014).

[29] H. Wu, CÓ Coileáin, M. Abid, O. Mauit, A. Syrlybekov, A. Khalid, H. Xu, R. Gatensby, J. J. Wang, H. Liu, and L. Yang, Spin-dependent transport properties of $\mathrm{Fe}_{3} \mathrm{O}_{4} / \mathrm{MoS}_{2} / \mathrm{Fe}_{3} \mathrm{O}_{4}$ junctions, Sci. Rep. 5, 15984 (2015).

[30] H. Zhang, M. Ye, Y. Wang, R. Quhe, Y. Pan, Y. Guo, Z. Song, J. Yang, W. Guoe, and J. Lu, Magnetoresistance in $\mathrm{Co} / 2 \mathrm{D} \mathrm{MoS}_{2} / \mathrm{Co}$ and $\mathrm{Ni} / 2 \mathrm{D} \mathrm{MoS}_{2} / \mathrm{Ni}$ junctions, Phys. Chem. Chem. Phys. 18, 16367 (2016).
[31] J. M. De Teresa, A. Barthelemy, A. Fert, J. P. Contour, F. Montaigne, and P. Seneor, Role of metal-oxide interface in determining the spin polarization of magnetic tunnel junctions, Science 286, 507 (1999).

[32] J. W. Chai, M. Yang, M. Callsen, J. Zhou, T. Yang, Z. Zhang, J. S. Pan, D. Z. Chi, Y. P. Feng, and S. J. Wang, Tuning contact barrier height between metals and $\mathrm{MoS}_{2}$ monolayer through interface engineering, Adv. Mater. Interfaces 4, 1700035 (2017).

[33] T. Garandel, R. Arras, X. Marie, P. Renucci, and L. Calmels, Electronic structure of the $\mathrm{Co}(0001) / \mathrm{MoS}_{2}$ interface and its possible use for electrical spin injection in a single $\mathrm{MoS}_{2}$ layer, Phys. Rev. B 95, 075402 (2017).

[34] M. Piquemal-Banci, R. Galceran, F. Godel, S. Caneva, M. B. Martin, R. S. Weatherup, P. R. Kidambi, K. Bouzehouane, S. Xavier, A. Anane, F. Petroff, A. Fert, S. M. M. Dubois, J. C. Charlier, J. Robertson, S. Hofmann, B. Dlubak, and P. Seneor, Insulator-to-metallic spin-filtering in 2D-magnetic tunnel junctions based on hexagonal boron nitride, ACS Nano 12, 4712 (2018).

[35] J. Pető, T. Ollár, P. Vancsó, Z. I. Popov, G. Z. Magda, G. Dobrik, C. Hwang, P. B. Sorokin, and L. Tapasztó, Spontaneous doping of the basal plane of $\mathrm{MoS}_{2}$ single layers through oxygen substitution under ambient conditions, Nat. Chem. 10, 1246 (2018).

[36] K. Dolui, I. Rungger, C. Das Pemmaraju, and S. Sanvito, Possible doping strategies for $\mathrm{MoS}_{2}$ monolayers: An ab initio study, Phys. Rev. B 88, 75420 (2013).

[37] S. El-Mahalawy and B. Evans, Temperature dependence of the electrical conductivity and hall coefficient in $2 \mathrm{H}-\mathrm{MoS}_{2}$, $\mathrm{MoSe}_{2}, \mathrm{WSe}_{2}$, and $\mathrm{MoTe}_{2}$, Phys. Status Solidi B 79, 713 (1977).

[38] S. El-Mahalawy and B. Evans, Pressure dependence of the electrical conductivity in $2 \mathrm{H}-\mathrm{MoS}_{2}$ and $2 \mathrm{H}-\mathrm{WSe}_{2}$, Phys. Status Solidi B 86, 151 (1978).

[39] M. Galbiati, A. Vecchiola, S. Mañas-Valero, J. CanetFerrer, R. Galceran, M. Piquemal-Banci, F. Godel, A. Forment-Aliaga, B. Dlubak, P. Seneor, and E. Coronado, A local study of the transport mechanisms in $\mathrm{MoS}_{2}$ layers for magnetic tunnel junctions, ACS Appl. Mater. Interfaces 10, 30017 (2018).

[40] J. P. Perdew, K. Burke, and M. Ernzerhof, Generalized Gradient Approximation Made Simple, Phys. Rev. Lett. 77, 3865 (1996).

[41] P. E. Blöchl, Projector augmented-wave method, Phys. Rev. B 50, 17953 (1994).

[42] G. Kresse and J. Joubert, From ultrasoft pseudopotentials to the projector augmented wave method, Phys. Rev. B 59, 1758 (1999).

[43] G. Kresse and J. Furthmüller, Efficient iterative schemes for $\mathrm{ab}$ initio total-energy calculations using a plane-wave basis set, Phys. Rev. B 54, 11169 (1996).

[44] S. Liang, H. Yang, P. Renucci, B. Tao, P. Laczkowski, S. Mc-Murtry, A. Djeffal, G. Wang, X. Marie, J.-M. George, S. Petit-Watelot, A. Djeffal, S. Mangin, H. Jaffrès, and Y. Lu, Electrical spin injection and detection in molybdenum disulfide multilayer channel, Nat. Commun. 8, 14947 (2017). 